\title{
Digital Signal Transformation and Computer Image Processing and Analysis Based on Multi-sensor Image Fusion
}

\author{
Fang $L i^{1, a}$ \\ ${ }^{1}$ Department of Computer, Wuhan Polytechnic, Wuhan, Hubei Province, China \\ alfanglf123@163.com
}

Keywords: Nanometer materials; sensor; image fusion; NSCT; PCNN.

\begin{abstract}
Image Fusion Technology is widely applied in various fields with the continuous development of image sensor technology. This study puts forward an image fusion algorithm based on Pulse Coupled Neural Network (PCNN) and Non Subsampled Contourlet Transform (NSCT). The algorithm does not make simultaneous use of PCNN on high frequency sub-band and low frequency sub-band. The fusion algorithm applied in fusion of low frequency sub-band coefficients is specifically designed for the high requirement on image definition of low frequency sub-band which can realize the fusion of local definition and marginal area energy. While the algorithm applied in fusion of high frequency sub-band coefficients takes bigger absolute values of coefficients, which is obtained from the highest level sub-band that can completely preserve marginal information showing images. For other hierarchies, PCNN fusion method which takes the spatial frequency as coefficient of connection is selected, so as to improve the visual features of fusion results.
\end{abstract}

\section{Introduction}

The concept of image fusion was put forward by scholars in the late 1970s (1). It is a high technology subject of artificial intelligence, image processing, sensors and computer technology. TONG Tao, et al. came up with an image fusion algorithm based on Non Subsampled Contourlet Transform (NSCT) so as to obtain excellent subjective visual effect and objective quantitative indicators (2). X Wang, et al. applied Multi-objective Particle Swarm Optimization (MOPSO) to find the optimal fusion parameters and solved collocation optimization problem of fusion parameters (3). A, et al. proposed a multi-source image fusion method based on revised Pulse Coupled Neural Network (PCNN) which overcame the shortages of image blurring and inadequate noise sensitivity which were commonly seen in traditional pixel level fusion methods and thus was especially suitable for situations where images could not be strictly matched (4).

This study puts forward a multisensor image fusion algorithm based on NSCT and PCNN transform, aiming to achieve better visual effect and quantitative indicators and therefore realize a better fusion of details of the source image.

\section{Image fusion algorithm based on combination of nsct and pcnn}

As shown in figure 1, fusion rule of NSCT multifocus image based on definition and PCNN is as follows: after NSCT multi-scale and multi-directional decomposition of the image, different rules was used for fusion processing on each sub-band coefficient. The final fusion image was obtained through NSCT inverse transformation. 


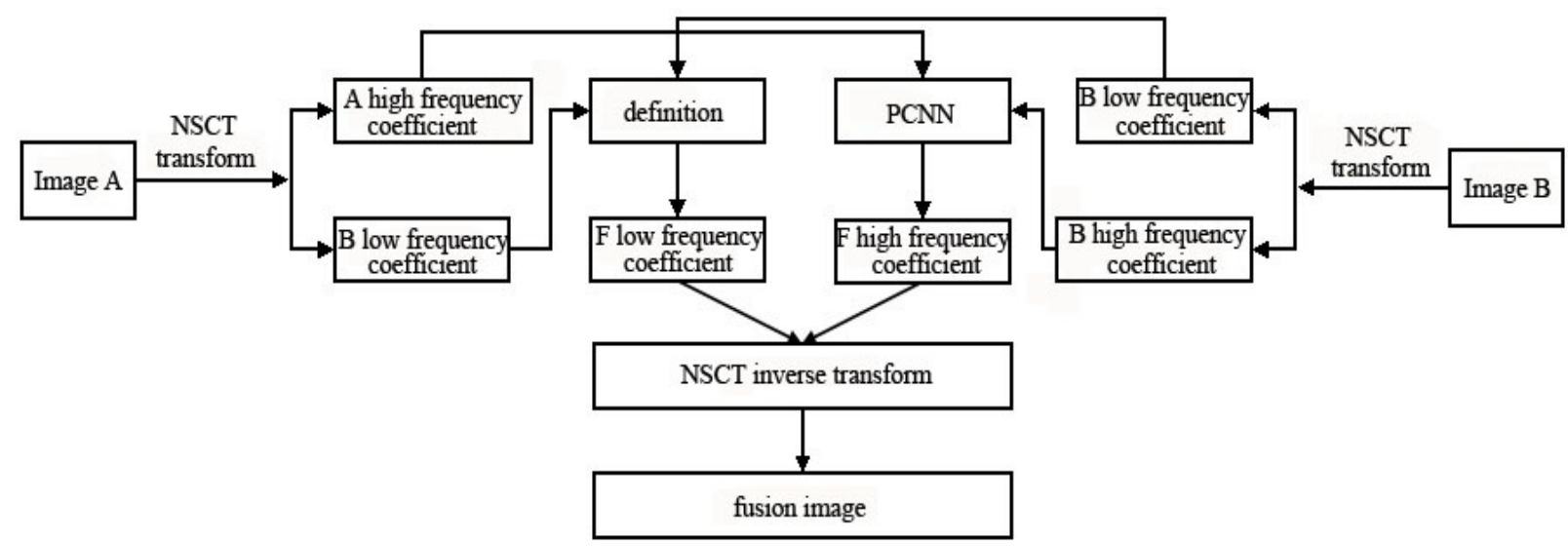

Fig. 1. Text algorithm flow chart

The specific fusion process is as follows:

Step 1: J level NSCT decomposition was carried out on image A and B and the all-order sub-band coefficients obtained were respectively $\left\{C_{j \mathrm{O}}^{A}(m, n), C_{j, l}^{A}(m, n)\right\}\left(j \geq j_{\mathrm{o}}\right)$ and $\mathrm{I}\left\{C_{j \mathrm{O}}^{B}(m, n), C_{j, l}^{B}(m, n)\right\}\left(j \geq j_{\mathrm{o}}\right)$

, of which, was the low frequency sub-band coefficient, was the band-pass sub-band coefficient on direction 1 under $\mathrm{j}$ dimension.

Step 2: for low frequency sub-band, selection scheme based on edge and clarity was applied and for high frequency sub-band, improved PCNN simplified model was applied. The coefficient of $\mathrm{F}$ obtained was $E_{A}(m, n)=\left(F_{1} * C_{j 0}^{A}\right)^{2}(m, n)+\left(F_{2} * C_{j 0}^{A}\right)^{2}(m, n)+\left(F_{3} * C_{j 0}^{A}\right)^{2}(m, n)$.

Step 3: NSCT inverse transformation was carried out and the final fusion image $\mathrm{F}$ was obtained.

Fusion Rule of Low Frequency Sub-band Coefficients. The main energy of the image is focused on the low-frequency part of the image. Low frequency sub-band fusion rules can lower image resolution and contrast. Therefore, this study puts forward a fusion rule based on a combination of reference (5) and (6) as follows:

Define a variable EA for low-frequency sub-band coefficients of source image A and it is called edge region energy:

$$
E_{A}(m, n)=\left(F_{1} * C_{j 0}^{A}\right)^{2}(m, n)+\left(F_{2} * C_{j 0}^{A}\right)^{2}(m, n)+\left(F_{3} * C_{j 0}^{A}\right)^{2}(m, n)
$$

Of which, * represents convolution,

$$
F_{1}=\left[\begin{array}{ccc}
-1 & -1 & -1 \\
2 & 2 & 2 \\
-1 & -1 & -1
\end{array}\right], F_{2}=\left[\begin{array}{lll}
-1 & 2 & -1 \\
-1 & 2 & -1 \\
-1 & 2 & -1
\end{array}\right], F_{3}=\left[\begin{array}{ccc}
-1 & 0 & -1 \\
0 & 4 & 0 \\
-1 & 0 & -1
\end{array}\right] \text {. }
$$

At the same time, define variable 5 on image $B$, and variable E reflects the marginal information on horizontal, vertical and diagonal direction of the image.

Local definition $\mathrm{G}(\mathrm{m}, \mathrm{n})$ was obtained according to the concept of definition.

$$
G(m, n)=\frac{1}{l^{2}} \sum_{m=-(l-1) / 2}^{(l-1) / 2} \sum_{n=-(l-1) / 2}^{(l-1) / 2} \sqrt{\left((\partial f(m, n) / \partial x)^{2}+(\partial f(m, n) / \partial y)^{2}\right) / 2}
$$

Of which, $\partial f(m, n) / \partial x$ and $\partial f(m, n) / \partial y$ represent respectively the differentials on $\mathrm{x}$ and $\mathrm{y}$ direction at point $(\mathrm{m}, \mathrm{n})$, denoted by change rate of coefficients of adjacent points. $l \times l$ represents size of the region which centered at point $(m, n)$. This study selects the $3 \times 3$ square region.

Discriminant factor after fusion can be obtained through the following equation:

$$
D(m, n)=E(m, n)\left(W_{1}+W_{2} G(m, n)\right)
$$


Of which, W1 and W2 are constants which are greater than 0 and are used for adjusting value of D. This study takes $\mathrm{W} 1=0, \mathrm{~W} 2=1$.

This study selects the scale coefficient with a higher $\mathrm{D}$ value as the low frequency sub-band coefficient of the fusion image, thus the edge and definition information can be maintained to the biggest extent. The fusion function was expressed as follows:

$$
C_{j 0}^{F}(m, n)=\left\{\begin{array}{l}
C_{j 0}^{A}(m, n), D_{A}(m, n) \geq D_{B}(m, n) \\
C_{j 0}^{B}(m, n), D_{A}(m, n)<D_{B}(m, n)
\end{array}\right.
$$

Fusion Rule of High- frequency Sub-band coefficients. The high frequency coefficients correspond to the detail information in the image (edge and texture), which is most sensitive to human eye recognition. As a result, this study applied the fusion method based on self-adaptive PCNN to process high frequency coefficients, and the fusion process was as follows:

Decomposition of $\mathrm{J}-1$ layer was carried out except dimension $\mathrm{J}$ at the highest layer. This study takes the high frequency coefficient as the trigger neuron of PCNN and thus spatial frequency after expansion is selected as the corresponding linking strength. The spatial frequency after expansion can be expressed as follows:

$$
S F=\sqrt{(R F)^{2}+(C F)^{2}+(M D F)^{2}+(S D F)^{2}}
$$

Among which, RF represents the line frequency; CF represents the column frequency; MDF represents the main diagonal frequency; SDF represents the secondary diagonal frequency.

$$
\begin{array}{ll}
R F=\sqrt{\frac{1}{M N} \sum_{m=1}^{M} \sum_{n=1}^{N}[I(m, n)-I(m, n-1)]^{2}} & C F=\sqrt{\frac{1}{M N} \sum_{m=2}^{M} \sum_{n=1}^{N}[I(m, n)-I(m-1, n)]^{2}} \\
M D F=\sqrt{\frac{1}{\sqrt{2} M N} \sum_{m=2}^{M} \sum_{n=1}^{N}[I(m, n)-I(m-1, n-1)]^{2}} & S D F=\sqrt{\frac{1}{\sqrt{2} M N} \sum_{m=2}^{M} \sum_{n=1}^{N-1}[I(m, n)-I(m-1, n+1)]^{2}}
\end{array}
$$

Linking intensity is expressed as follows:

$$
\beta=\eta S F(m, n)
$$

Of which, $\eta$ is a constant which is greater than 0 , and it is used for adjusting the value of $\beta$.

Taking the high frequency sub-band coefficients as the input of PCNN, diagram of duration of ignition $\mathrm{Y}_{\mathrm{A}}$ and $\mathrm{Y}_{\mathrm{B}}$ of high frequency sub-band coefficients of image $\mathrm{A}$ and $\mathrm{B}$ can be obtained.

$$
C_{j, l}^{F}(m, n)=\left\{\begin{array}{l}
C_{j, l}^{A}(m, n), Y_{A}(m, n) \geq Y_{B}(m, n) \\
C_{j, l}^{B}(m, n), Y_{A}(m, n)\left\langle Y_{B}(m, n)\right.
\end{array}\right.
$$

In order to decompose high frequency coefficients of point dimension $\mathrm{J}$ of the highest layer, this study selects the method which takes the bigger absolute values.

$$
R F=\sqrt{\frac{1}{M \times N} / \sum_{x=0}^{M-1} \sum_{y=1}^{N-1}[F(x, y)-F(x, y-1)]^{2}} \quad C F=\sqrt{\frac{1}{M \times N} / \sum_{x=1}^{M-1} \sum_{y=0}^{N-1}[F(x, y)-F(x-1, y)]^{2}}
$$

\section{Evaluation Standard of Image Fusion}

Subjective Evaluation Method. Subjective evaluation method (7) is also known as Visual Observation. When judging on quality of fusion images, experimenters should observe and evaluate the results with naked eyes. However, human eyes are unable to detect subtle changes, which results in the inaccuracy and subjectivity of the evaluation. Therefore, objective evaluation should be applied to form a comprehensive evaluation system with a co-existence of subjectivity and objectivity.

Objective Evaluation Method. Information entropy, spatial frequency and average gradient are popular objective evaluation indexes. Since this objective evaluation applies formulas, the indexes are relatively standard. 
Information Entropy. Information Entropy is an important indicator of objective evaluation. Measurement of information richness can be realized through comparing values of image information entropy. The image entropy (8) is defined as follows:

$$
E=-\sum_{i=0}^{L-1} p(i) \log _{2} p(i)
$$

Of which, L represents the total number of gray level; $p(i)$ is the probability of appearance of pixel whose gray level is $i$.

Spatial Frequency. Spatial frequency (SF) includes column frequency (CF) and row frequency (RF), which are defined as follows:

$$
R F=\sqrt{\frac{1}{M \times N} / \sum_{x=0}^{M-1} \sum_{y=1}^{N-1}[F(x, y)-F(x, y-1)]^{2}} \quad C F=\sqrt{\frac{1}{M \times N} / \sum_{x=1}^{M-1} \sum_{y=0}^{N-1}[F(x, y)-F(x-1, y)]^{2}}
$$

Of which, $\mathrm{F}(\mathrm{x}, \mathrm{y})$ is the gray value; $\mathrm{M}$ and $\mathrm{N}$ are the number of rows and columns respectively. The final spatial frequency is the mean square root of RF and CF, as follows:

$$
S F=\sqrt{R F^{2}+C F^{2}}
$$

The greater the value of SF, the better the fusion effect can be.

Average Gradient. Image definition can be reflected by the average gradient of the image. The average gradient of the image (9) is defined as follows:

$$
A V G=\frac{1}{M \times N} \sum_{x=0}^{M-1} \sum_{y=0}^{N-1} \sqrt{\frac{G_{x}^{2}(x, y)+G_{y}^{2}(x, y)}{2}}
$$

Of which, $\mathrm{Gx}(\mathrm{x}, \mathrm{y})$ and $\mathrm{Gy}(\mathrm{x}, \mathrm{y})$ are respectively the gradient on $\mathrm{x}$ and $\mathrm{y}$ direction of pixel point (x, $\mathrm{y})$; $\mathrm{M}$ and $\mathrm{N}$ are the number of columns and rows. The less the image level, the smaller the average gradient will be; on the contrary, the higher the image definition, the bigger the average gradient will be.

\section{Experimental results and performance evaluation}

In order to verify the validity and superiority of this method, registered multifocus image $a$ and $b$ were applied based on matlab7.0. Besides, contrast experiment was carried out respectively with Wavelet Transform, NSCT and PCNN method and objective evaluation was made on the results, as shown in figure 2.

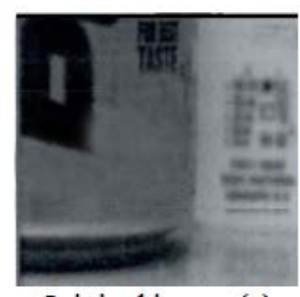

Original image (a)

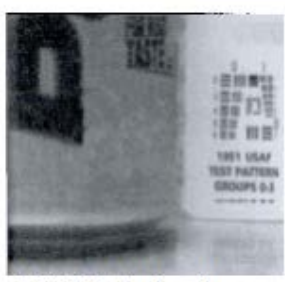

NSCT fusion image

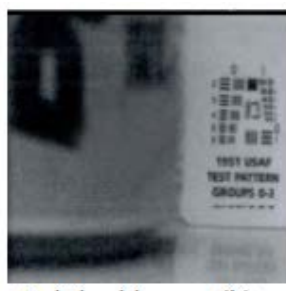

Original image (b)

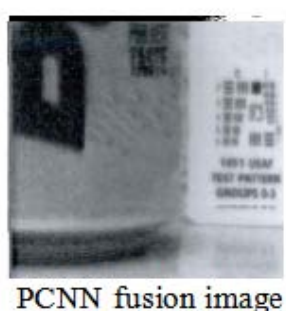

PCNN fusion image

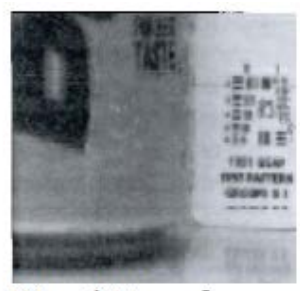

Wavelet transform fusion image

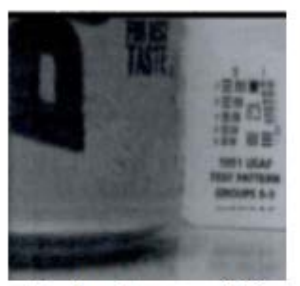

Fusion image of the method applied in this study

Fig. 2. image fusion results 
Objective Evaluation of Image Fusion. This study applied three evaluation parameters including information entropy, spatial frequency and average gradient and carried out a quantitative comparison on the image fusion performance between the following methods, as shown in table 1:

\begin{tabular}{cccc}
\hline & Information entropy & Spatial frequency & Average gradient \\
\hline Wavelet transform fusion image & 7.1084 & 11.6615 & 4.6742 \\
\hline NSCT fusion image & 7.0986 & 12.7813 & 5.1057 \\
\hline PCNN fusion image & 7.4865 & 14.3536 & 5.3946 \\
\hline Fusion image of the method in this study & 7.1213 & 13.7093 & 5.3708 \\
\hline
\end{tabular}

Table 1 showed that most of the indicators of the method proposed in this study were superior to those of comparing fusion algorithm. The greater the value of information entropy, spatial frequency and average gradient, the better the quality of image would be. However, due to the simple application of the bionic algorithm, much noise was introduced by PCNN method which resulted in much difference between the source image and the fusion image with a big cross entropy value. Therefore, the method applied in this study is the best of the four methods.

\section{Conclusion}

This study put forward a combined approach to realize the fusion of the source image based on PCNN and NSCT. The experimental results showed that quality of the obtained fusion image through the method selected in this study was superior to that of Laplacian pyramid method, Wavelet Transform and traditional method based on NSCT transform. The multi-focus image fusion algorithm proposed in this study can get the fusion image with all clear goals, which improves the observability and use value of the image and better retains the useful information and detail characteristics of the source image.

\section{Acknowledgement}

Fund of Hunan provincial education department (15A171), Fund of hunan provincial education department (13C842).

\section{References and Notes}

[1] H. Li, B.S. Manjunath, S.K. Mitra. Multisensor Image Fusion Using the Wavelet Transform. Graphical Models and Image Processing, 57(3):235-245, 1995.

[2] T. Tao, Y. Guang, T. Hai feng, REN Chun ying. Multi-Sensor Image Fusion Algorithm Based on NSCT. Geography and Geo-Information Science, 29(2): 22-308, 2013.

[3] X. Wang, Z. Fang sheng, M. Xin, L. Xu qing. Multi-sensor Image Fusion Based on Multi-objective Particle Swarm Optimization Algorithm. Opto-Electronic Engineering, 39(6):102-110, 2012.

[4] H. Wang, Q. Yang, R. Li. Tunable-Q contourlet-based multi-sensor image fusion. Signal Processing, 93(7):1879- 1891, 2013.

[5] H. M. Wang, K. Zhang, Y. J. Li. Image fusion algorithm based on wavelet transform. Infrared \& Laser Engineering, 34(3): 328-332, 2005.

[6] A. J. Shah, R. Makwana, S. B. Gupta, et al. Single Image Super-Resolution via Non Sub-sample Contourlet Transform based Learning and a Gabor Prior. International Journal of Computer Applications, 64(18):32-38, 2013. 
[7] L. Xin liang, Z. Shu he, K. Chang qing, G. Kai yu. The Study of Methods of Quantitative Evaluation on Remote Sensing Image Fusion and Actualization. Remote Sensing Technology \& Application, 22(3):460-465, 2007.

[8] D. Connah, M. Samuelw, G. D. Finlayson. Spectral Edge Image Fusion: Theory and Applications. Lecture Notes in Computer Science, 8693:65-80, 2014.

[9] M. Ding, L. Wei, B. Wang. Research on fusion method for infrared and visible images via compressive sensing. Infrared Physics \& Technology, 57(2):56-67, 2013. 